\title{
APPENDIX 6
}

\section{Invasive Plant Species}

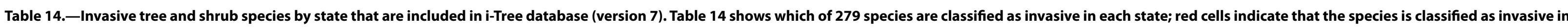

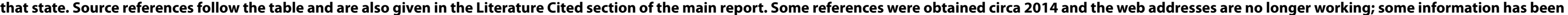
relocated to alternative sites.

State

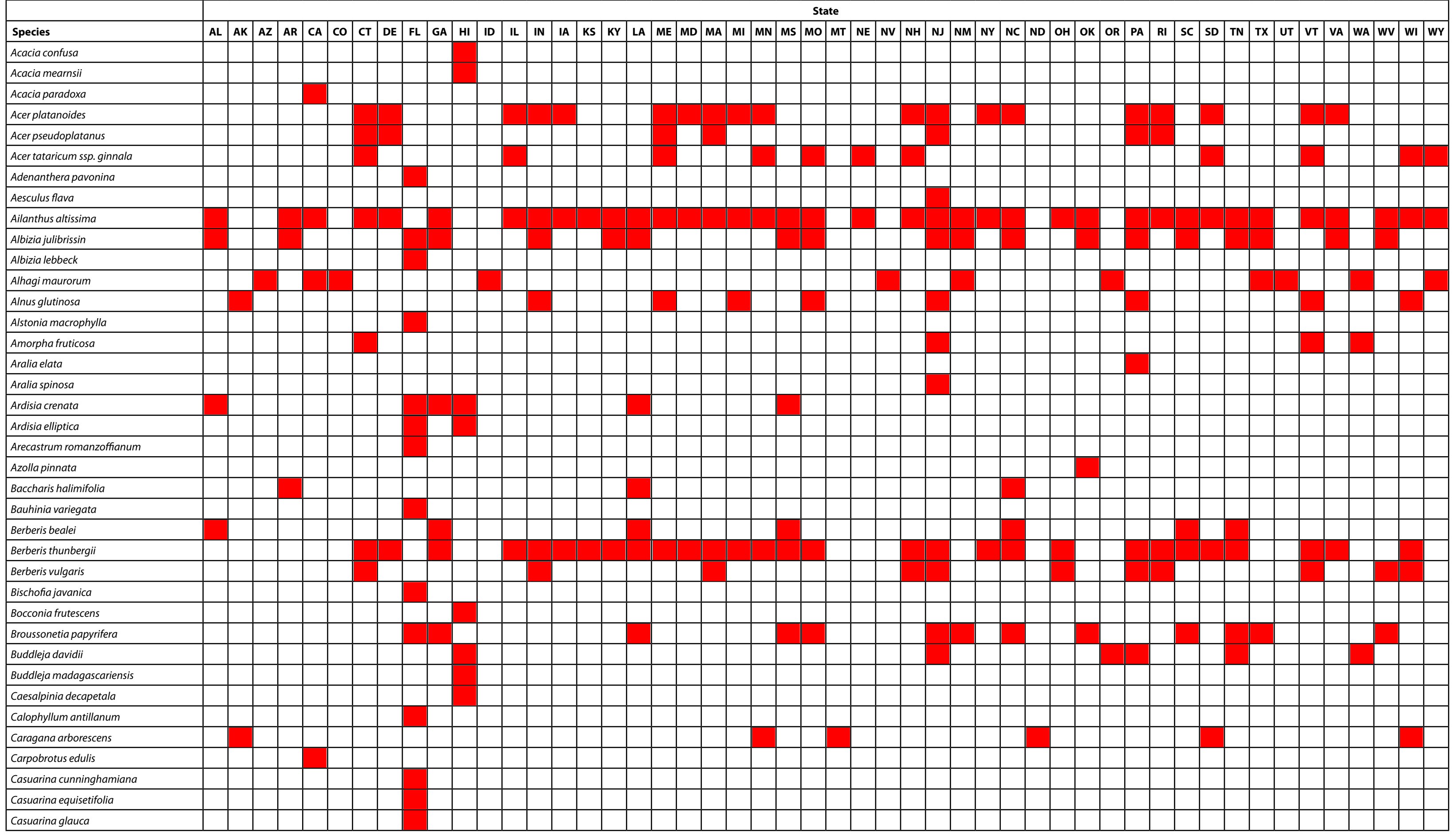

Appendix 6 (https://doi.org/10.2737/NRS-GTR-200-2021-Appendix6) in: Nowak, David J. 2021. Understanding i-Tree: Summary of programs and methods. Gen. Tech. Rep. NRS-200-2021. Madison, WI: U.S. Department of Agriculture, Forest Service, Northern Research Station. 100 p. [plus 14 appendixes]. https://doi.org/10.2737/NRS-GTR-200-2021. 


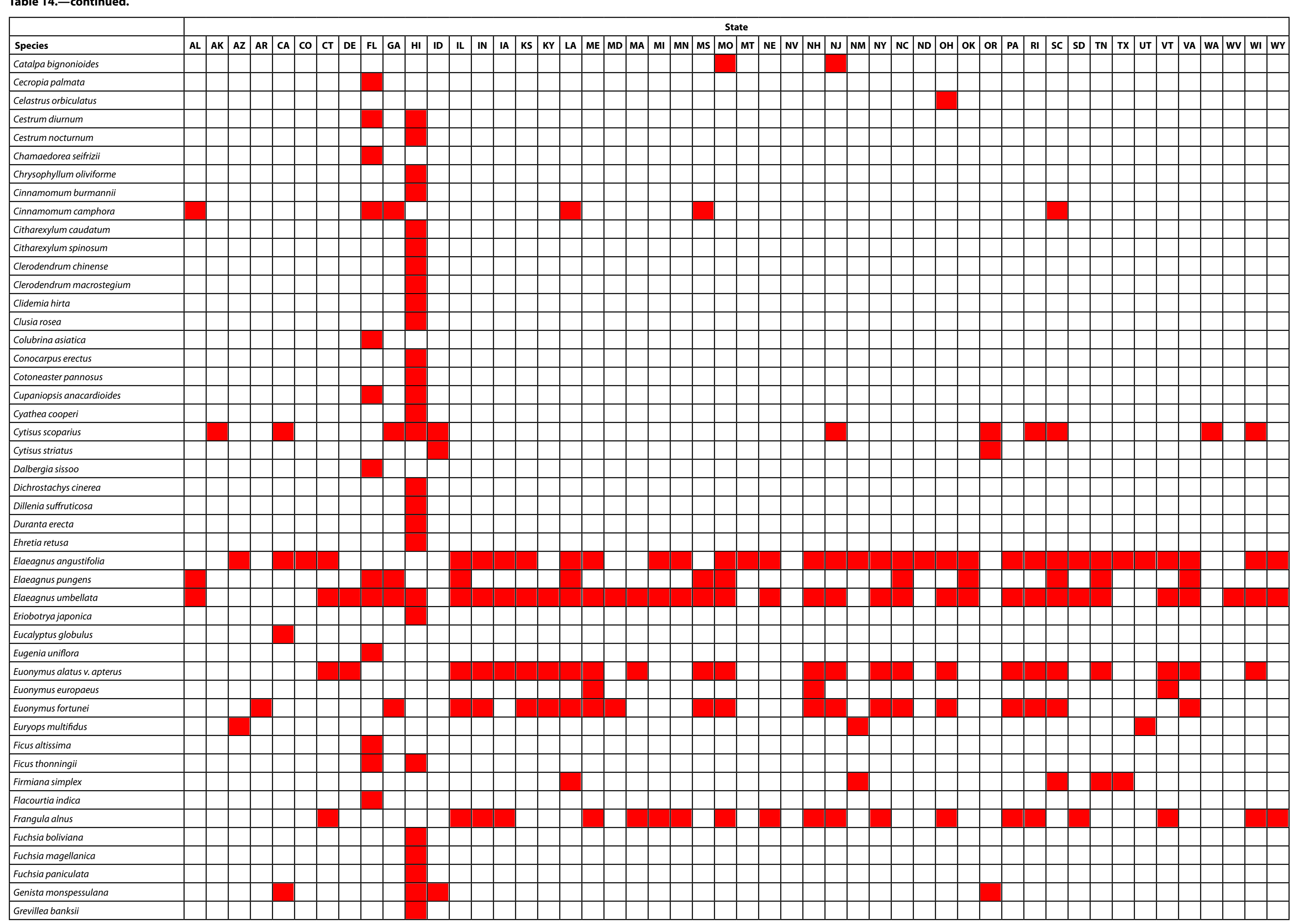


Table 14.-continued.

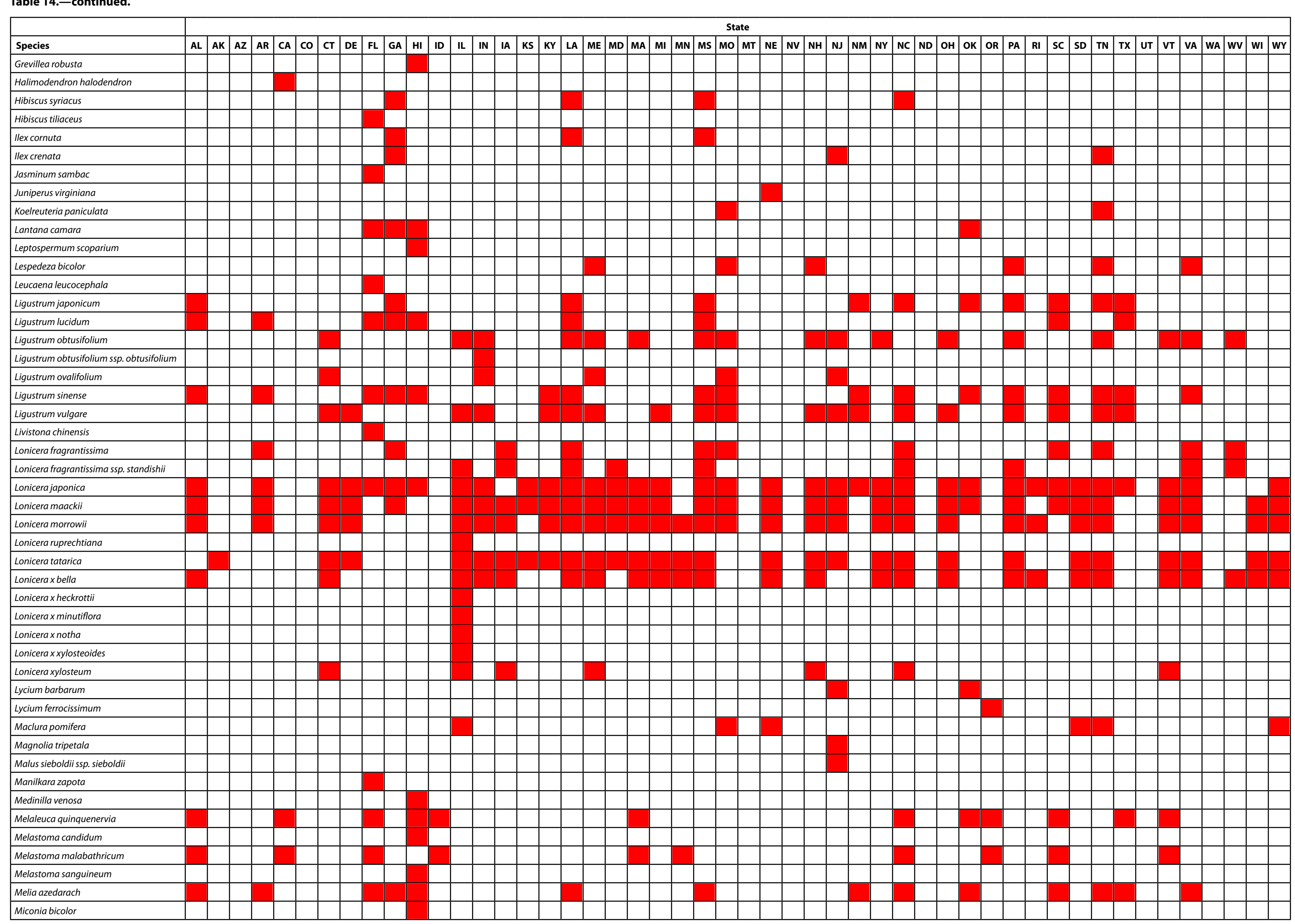


Table 14.-Continued.

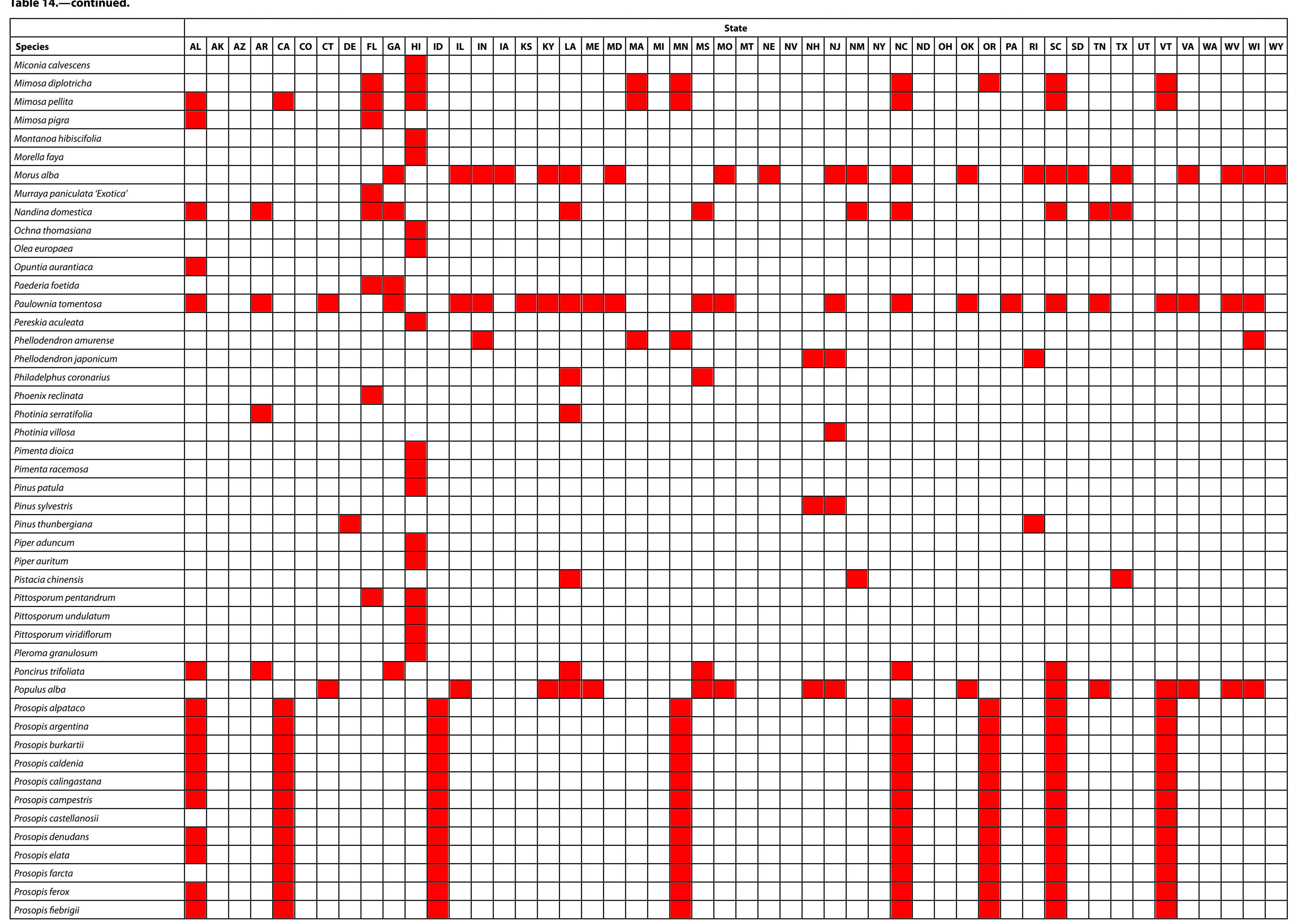


Table 14.-Continued.

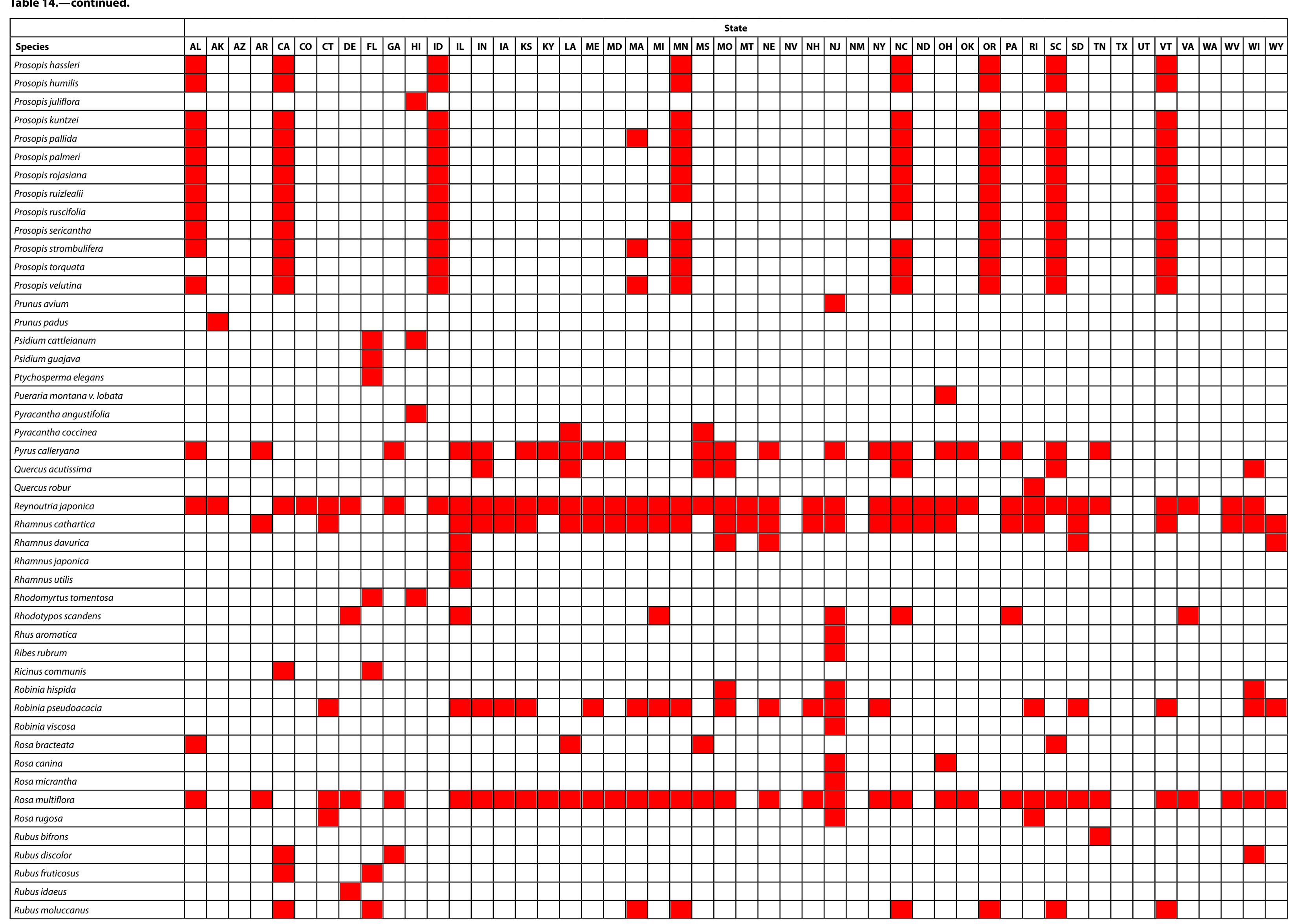


Table 14.-Continued.

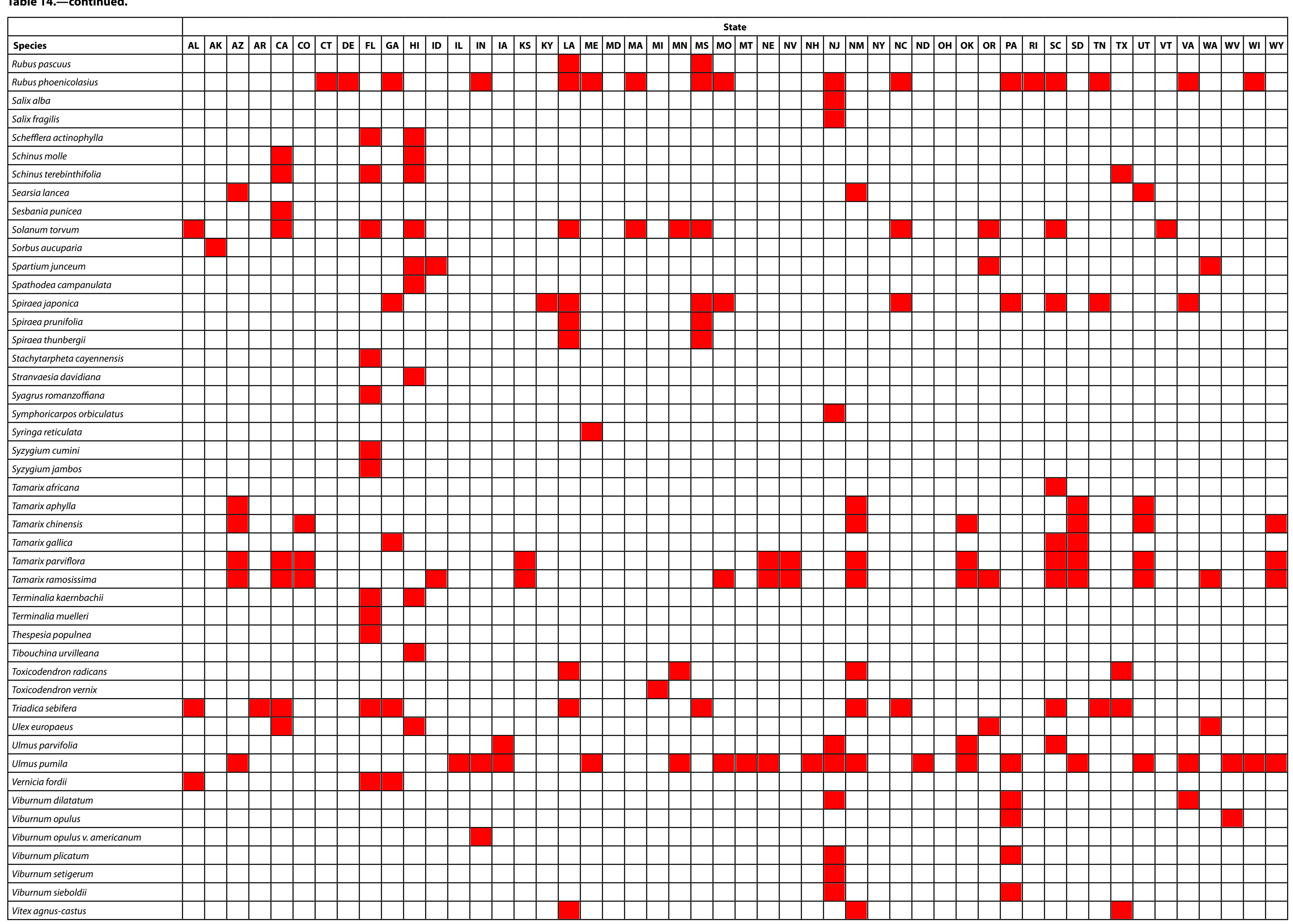




\section{Table 14.-continued.}

\begin{tabular}{|c|c|c|c|c|c|c|c|c|c|c|c|c|c|c|c|c|c|c|c|c|c|c|c|c|c|c|c|c|c|c|c|c|c|c|c|c|c|c|c|c|c|c|c|c|c|c|c|c|c|c|c|}
\hline & \multicolumn{51}{|c|}{ State } \\
\hline Species & AL & AK & $A Z$ & AR & $\mathrm{CA}$ & cc & c & D & & $\mathrm{FL}$ & GA & HI & ID & IL & IN & IA & KS & KY & LA & ME & MD & MA & MI & MN & MS & mo & MT & NE & NV & $\mathrm{NH}$ & NJ & NM & NY & NC & ND & $\mathrm{OH}$ & OK & OR & $\mathbf{P A}$ & $\mathbf{R I}$ & SC & SD & $\mathrm{TN}$ & $T \mathrm{TX}$ & UT & VT & VA & WA & wv & wI & wY \\
\hline Vitex trifolia & & & & & & & & & & & & & & & & & & & & & & & & & & & & & & & & & & & & & & & & & & & & & & & & & & & \\
\hline Vitex trifolia ssp. & & & & & & & & & & & & & & & & & & & & & & & & & & & & & & & & & & & & & & & & & & & & & & & & & & & \\
\hline Washingtonia rc & & & & & & & & & & & & & & & & & & & & & & & & & & & & & & & & & & & & & & & & & & & & & & & & & & & \\
\hline Wisteria floribur & & & & & & & & & & & & & & & & & & & & & & & & & & & & & & & & & & & & & & & & & & & & & & & & & & & \\
\hline Wisteria sinensis & & & & & & & & & & & & & & & & & & & & & & & & & & & & & & & & & & & & & & & & & & & & & & & & & & & \\
\hline Zelkova serrata & & & & & & & & & & & & & & & & & & & & & & & & & & & & & & & & & & & & & & & & & & & & & & & & & & & \\
\hline
\end{tabular}




\section{References}

Some of the listed web addresses are no longer functional but were active at the time the invasive tree and shrub database was developed for i-Tree (version 7).

Alabama Invasive Plant Council. 2007. 2007 plant list. Athens, GA: Center for Invasive Species and Ecosystem Health, Southeast Exotic Pest Plant Council. http://www.se-eppc.org/ alabama/2007plantlist.pdf.

Alaska National Heritage Program. 2014. Non-Native plant data. Anchorage, AK: University of Alaska Anchorage. http:// aknhp.uaa.alaska.edu/botany/akepic/non-native-plant-specieslist/\#content.

Arizona Wildland Invasive Plant Working Group. 2005. Invasive non-native plants that threaten wildlands in Arizona. Phoenix, AZ: Southwest Vegetation Management Association. https:// www.swvma.org/wp-content/uploads/Invasive-Non-NativePlants-that-Threaten-Wildlands-in-Arizona.pdf.

Ashigh, J. Wanstall, J., Sholedice, F. 2010. Troublesome weeds of New Mexico. La Cruces, NM: New Mexico State University, College of Agricultural, Consumer and Environmental Sciences, Cooperative Extension Service. 104 p. https://www.nmda.nmsu. edu/wp-content/uploads/2012/04/troublesome weeds nm.pdf.

California Invasive Species Advisory Committee. 2010. The California invasive species list. Sacramento, CA: Invasive Species Council of California. http://www.iscc.ca.gov/docs/ CaliforniaInvasiveSpeciesList.pdf.

Center for Invasive Species and Ecosystem Health. 2009. Illinois invasive plant list. Athens, GA: Center for Invasive Species and Ecosystem Health. Invasive.org. http://www.invasive.org/species/.

City of Austin, TX. 2013. Central Texas invasive plants. https:// www.austintexas.gov/sites/default/files/files/Watershed/ invasive/2013 Invasives guide small.pdf.

Colorado Weed Management Association. 2012. Colorado's noxious weed list. Arvada, CO: Colorado Weed Management Association. https://cwma.org/weed-information/weed-list/.

Connecticut Invasive Plant Working Group. 2013. Invasive plant list. Storrs, CT: University of Connecticut. http://cipwg.uconn. $\underline{\text { edu/invasive plant list/. }}$

Cusack, C.; Plumb, S.; Prince, D. 2011. Developing invasive plant outreach and management projects. Montpelier, VT: The Nature Conservancy (see Table 1.2).

Cygan, D. 2011. New Hampshire guide to invasive upland plant species, 3rd edition. Concord, NH: Department of Agriculture, Division of Plant Industry. http://www.agriculture.nh.gov/ publications-forms/documents/upland-invasive-species.pdf.
Delach, A. n.d. Invasive species in Utah. https://defenders.org/ sites/default/files/publications/utah.pdf.

Florida Exotic Pest Plant Council. 2007. Florida EPPC’s 2007 invasive plant species list. Athens, GA: Center for Invasive Species and Ecosystem Health, Southeast Exotic Pest Plant Council. https://www.fleppc.org/list/2007/07list.htm.

Forest Invasive Plants Resource Center. 2006. Iowa. U.S Department of Agriculture, Forest Service, Northeastern Area State and Private Forestry. http://na.fs.fed.us/spfo/invasiveplants/ states/ia.asp.

Georgia Exotic Pest Plant Council. 2006. List of non-native invasive plants in Georgia. Athens, GA: Center for Invasive Species and Ecosystem Health, Southeast Exotic Pest Plant Council. https://www.gaeppc.org/list/.

Grafton, E. 2003. Dirty dozen. Charleston, WV: West Virginia Division of Natural Resources, Wildlife Resources. http://www. wvdnr.gov/Wildlife/DirtyDozen.shtm.

Hawaii State Alien Species Coordinator. 2012. Hawaii's most invasive horticultural plants. Honolulu, HI: Department of Land and Natural Resources, Division of Forestry and Wildlife. http://www.gchonolulu.org/FlowerShow2012/ HORTICULTURAL\%20PLANTS\%20Invasive.PDF. This website is no longer available; a similar, but not exact, list can now be found at: http://www.hear.org/hortweeds/

Idaho Association of Weed Superintendents and University of Idaho Extension Office. 2020. Invasive species of Idaho. http:// invasivespecies.idaho.gov/terrestrial-plants.

Illinois Department of Natural Resources. 2020. Invasive species. https://www2.illinois.gov/dnr/conservation/IWAP/Pages/ InvasiveSpecies.aspx.

Illinois Department of Natural Resources. 2020. Invasive species campaign. https://www2.illinois.gov/dnr/conservation/IWAP/ Documents/InvasiveSpecies.pdf.

Indiana Cooperative Agricultural Pest Survey. 2007. Indiana's "Most Unwanted" invasive plant pest list - FY 2007. West Lafayette, IN: Purdue University, Purdue Extension Entomology. http://www.extension.entm.purdue.edu/CAPS/downloads/ IndianaInvasivePlantPestList.pdf.

Indiana Department of Natural Resources. 2020. Invasive plant species. https://www.in.gov/dnr/naturepreserve/4736.htm.
Indiana Invasive Species Council. 2020. Official IISC invasive plant list. https://www.entm.purdue.edu/iisc/invasiveplants.html.

Invasive Native Plant Society of New Jersey. 2004. Appendix to policy directive 2004-02 invasive nonindigenous plant species. https://www.nj.gov/dep/commissioner/policy/pdir2004-02.htm.

Iowa Department of Natural Resources. Invasive plants. Des Moines, IA: Department of Natural Resources, Forestry Bureau. http://www.iowadnr.gov/Environment/Forestry/ForestHealth/ InvasivePlants.aspx.

Jardine, J.; Witsell, T. n.d. Working list of non-native invasive plant species of concern to natural areas in Arkansas. Arkansas Native Plant Society. [URL no longer available.]

Kansas Forest Service. 2020. Invasive species. https://www. kansasforests.org/forest_health/invasivespecies.html.

Kansas Native Plant Society. 2006. Invasive plants of Kansas. Lawrence, KS: University of Kansas, R.L. McGregor Herbarium. http://www.kansasnativeplantsociety.org/invasive plants.htm.

Kentucky Exotic Pest Plant Council. 2013. Kentucky exotic pest plant council. Athens, GA: Center for Invasive Species and Ecosystem Health, Southeast Exotic Pest Plant. http://www.seeppc.org/ky/list.htm.

Ling, H. 2003. Invasive plant species. Native Plant Society of New Jersey. http://www.npsnj.org/articles/invasive plant species. $\underline{\mathrm{html}}$.

Maine Legislature. 2000. An act to prevent the spread of invasive aquatic plants. Augusta, ME: Maine State Legislature, Office of the Revisor of Statutes. http://www.mainelegislature.org/ros/ LOM/LOM119th/5Pub701-750/5Pub701-750-21.htm.

Maine Department of Agriculture, Conservation, and Forestry. Various dates. Maine invasive plant fact sheets. Augusta, ME Department of Agriculture, Conservation, and Forestry, Maine Natural Areas Program. http://www.maine.gov/dacf/mnap/ features/invasive plants/invsheets.htm.

Maryland Invasive Species Council. 2014a. Invasive species of concern in Maryland: terrestrial plants. http://www. mdinvasivesp.org/list terrestrial plants.html.

Maryland Invasive Species Council. 2014b. Invasive species of concern in Maryland: aquatic plants. http://www.mdinvasivesp. org/list aquatic plants.html. 
Massachusetts Invasive Plant Advisory Group. 2005. The evaluation of non-native plant species for invasiveness in Massachusetts. http://www.massnrc.org/mipag/docs/MIPAG FINDINGS FINAL 042005.pdf.

McAvoy, W.A. 2001. Invasive plant species in Delaware. Dover, DE: Department of Natural Resources and Environmenta Control. http://www.dnrec.state.de.us/fw/invasive.htm.

Michigan Natural Features Inventory. 2014. Michigan invasive plant species accounts. Lansing, MI: Michigan State University Extension, Michigan Natural Features Inventory. http://mnfi.anr. msu.edu/invasive-species/factsheets.cfm.

Minnesota Department of Natural Resources. 2014a. Invasive terrestrial plants. http://www.dnr.state.mn.us/invasives/ terrestrialplants/index.html.

Minnesota Department of Natural Resources. 2014b. Invasive aquatic plants. http://www.dnr.state.mn.us/invasives/ aquaticplants/index.html.

Mississippi Exotic Pest Plant Council. 2014. Noteworthy exotic plant species of Mississippi. Athens, GA: Center for Invasive Species and Ecosystem Health, Southeast Exotic Pest Plant. http://www.se-eppc.org/mississippi/MS EPPCList Ranking Guidelines.pdf.

Missouri Botanical Garden. 2002. Missouri exotic pest plants. St. Louis, MO: Missouri Botanical Garden. http://www.mobot.org/ mobot/research/mepp/alphalist.shtml.

Missouri Department of Conservation. 2020. Invasives. https:// nature.mdc.mo.gov/status/.

Nebraska Invasive Species Advisory Council. Invasive plants of Nebraska. https://neinvasives.com/ecoregions/all.

New Hampshire Department of Agriculture. 2012. Prohibited invasive plant species rule. Concord, $\mathrm{NH}$ : Department of Agriculture, Division of Plant Industry. http://www.agriculture. nh.gov/publications-forms/documents/prohibited-invasivespecies.pdf.

New Hampshire Department of Agriculture. 2014. List of restricted species. Concord, NH: Department of Agriculture, Division of Plant Industry. http://www.agriculture.nh.gov/publicationsforms/documents/restricted-invasive-species.pdf.

New Mexico Department of Agriculture. 2020. Noxious weed information. https://www.nmda.nmsu.edu/nmda-homepage/ divisions/apr/noxious-weed-information/.
New York State Department of Environmental Conservation. 2020. Invasive species regulations. https://www.dec.ny.gov/ animals/99141.html.

New York State Department of Environmental Conservation. Interim invasive species plant list. http://www.dec.ny.gov/ animals/65408.html.

North Carolina Native Plant Society. 2010. Invasive exotic species list. Greensboro, NC: North Carolina Native Plant Society. http:// www.ncwildflower.org/invasives/list.htm.

North Dakota Department of Agriculture. 2020. Noxious weeds https://www.nd.gov/ndda/plant-industries/noxious-weeds.

North Dakota Department of Agriculture. Catalogue of species. [URL no longer available.].

Northwest Illinois Forestry Association. 2020. Exotic invasive plants. http://www.nifatrees.org/Invasive-Species.

Ohio Department of Agriculture. 2018. Invasive plants. https:// agri.ohio.gov/wps/portal/gov/oda/divisions/plant-health/ invasive-pests/invasive-plants.

Ohio Division of Natural Areas and Preserves. 2000. Ohio's invasive plant species. Columbus, $\mathrm{OH}$ : Department of Natural Resources, Division of Natural Areas and Preserves.

OK Invasives. 2020. Invasive species to keep an eye on! https:// www.okinvasives.org/watch-list-1.

Oklahoma Native Plant Society; Oklahoma Biological Survey; OSU Natural Resource Ecology and Management. Oklahoma nonnative invasive plant species. Norman, OK: Oklahoma Invasive Plant Council. [URL no longer available.]

Pennsylvania Department of Conservation and Natural Resources. Invasive plants in Pennsylvania. Harrisburg, PA: Department of Conservation and Natural Resources. http://www.dcnr.state pa.us/cs/groups/public/documents/document/denr 20026634. $\mathrm{pdf}$

Pernas, T.; Giardina, D.; Duquesnel, J.; Franck, A.; Hammer, R.L. et al.]. 2019. Florida Exotic Pest Plant Council's 2019 list of invasive plant species. http://bugwoodcloud.org/CDN/fleppc/ plantlists/2019/2019_Plant_List_ABSOLUTE_FINAL.pdf.

Rhode Island Invasive Species Council; Rhode Island Natural History Survey. 2001. Invasives: list. http://rinhs.org/wpcontent/uploads/2011/10/RIISC 2001list_wlogos.pdf.

Smith, C. 2008. Invasive exotic plants of North Carolina Charlotte, NC: North Carolina Department of Transportation.
http://www.se-eppc.org/northcarolina/NCDOT Invasive Exotic Plants.pdf

South Carolina Exotic Pest Plant Council. 2008. Invasive species list 2008. Athens, GA: Center for Invasive Species and Ecosystem Health. http://www.invasive.org/species/list.cfm?id=27.

South Dakota Department of Agriculture. 2020. State noxious weed \& pest list. https://sdda.sd.gov/ag-services/weed-and-pestcontrol/weed-pest-control/sd-state-noxious-weed-declared-pestlist-and-distribution-maps/.

State of Michigan. 2020. Michigan invasive species. https://www. michigan.gov/invasives/0,5664,7-324-68002 $7124073850---, 00$ $\underline{\text { html. }}$

Stoddard, S.W.; Johnson, W.S.; Wilson, R.E. 2013. Invasive plants in Nevada: An identification handbook. SP 96-03. Reno, NV: University of Nevada, Cooperative Extension. http://www.unce. unr.edu/publications/files/ag/other/sp9603.pdf.

Tennessee Exotic Pest Plant Council. 2009. TN-EPPC invasive exotic pest plants in Tennessee. Fairview, TN: Tennessee Exotic Pest Plant Council. https://sites.google.com/site/tnarboretum/ Home/tn-eppc-invasive-exotic-pest-plants-in-tennessee.

Tennessee Invasive Pant Council. 2020. TN-IPC 2017 list revision. https://www.tnipc.org/revised-list-of-invasive-plants/.

TexasInvasives.org. 2020. Invasives database. https://texasinvasives. org/plant database/coa results.php?offset $=12$.

USDA Natural Resources Conservation Service, Plants Database. 2017. Introduced, invasive, and noxious plants [for Oregon]. https://plants.usda.gov/java/noxious?.rptType=State\&statefi ps $=41$.

USDA Natural Resources Conservation Service. 2017. Introduced, invasive, and noxious plants [for Washington]. https://plants. usda.gov/java/noxious? rptType=State\&statefips=53.

USDA Natural Resources Conservation Service. 2017. Introduced, invasive, and noxious Plants [for West Virginia]. https://plants. usda.gov/java/noxious? rptType $=$ State\&statefips $=54$.

Vermont Invasive Exotic Plant Committee. 2005. Invasive species watch list for Vermont. https://fpr.vermont.gov/sites/fpr/files/ Quarantine WatchListUpdate-2017_November.pdf.

Virginia Department of Conservation and Recreation. 2009. Invasive alien plant species of Virginia. Richmond, VA Department of Conservation and Recreation, Division of Natura Heritage. [Original URL unavailable.] Updated list: https://www. dcr.virginia.gov/natural-heritage/document/nh-invasive-plantlist-2014.pdf. 
Watershed Protection Development Review. N.d. Central Texas invasive plants. Austin, TX: City of Austin, Watershed Protection Development Review.

Wisconsin Department of Natural Resources. 2018. Terrestrial invasive species. http://dnr.wi.gov/topic/Invasives/species.asp?fi $\underline{\text { terBy}}=$ Terrestrial\&filterVal $=\mathrm{Y}$.
WVDNR Natural Heritage Program. 2009. Invasive plant species of West Virginia. http://www.wvdnr.gov/wildlife/Handout\%20 Invasive\%20Plants\%20of\%20WV\%202009.pdf.

Wyoming Weed and Pest Council. 2019. State designated noxious weeds. https://wyoweed.org/noxious-species/listed-species/statedesignated-noxious-weeds/. 